\title{
Focus. Intertextualité et exogenèse devant les tribunaux
}

L'exogenèse à la barre

\section{Emmanuel Pierrat}

\section{OpenEdition}

\section{Journals}

Édition électronique

URL : https://journals.openedition.org/genesis/5636

DOI : $10.4000 /$ genesis.5636

ISSN : 2268-1590

Éditeur :

Presses universitaires de Paris Sorbonne (PUPS), Société internationale de génétique artistique littéraire et scientifique (SIGALES)

\section{Édition imprimée}

Date de publication : 15 décembre 2020

Pagination : $45-50$

ISBN : 979-10-231-0704-3

ISSN : $1167-5101$

\section{Référence électronique}

Emmanuel Pierrat, "Focus. Intertextualité et exogenèse devant les tribunaux », Genesis [En ligne], 51 | 2020, mis en ligne le 20 décembre 2021, consulté le 07 février 2022. URL : http:// journals.openedition.org/genesis/5636 ; DOI : https://doi.org/10.4000/genesis.5636 


\section{Intertextualité et exogenèse devant les tribunaux ou L'exogenèse à la barre}

Emmanuel Pierrat

Avant, en littérature, quand il y avait un clin d'œil, on applaudissait, aujourd'hui on tombe à bras raccourcis sur l'auteur [...]. Et les emprunts, cela devient un crime, un blasphème [...] La littérature ne s'écrit pas ex-nihilo, les auteurs se nourrissent les uns des autres et l'ont toujours fait. L'intertextualité, c'est un classique de la littérature $[\ldots]^{1}$.

Ainsi s'est défendu, en 2011, le journaliste Joseph Macé-Scaron, alors directeur adjoint de Marianne et directeur du Magazine littéraire. Il était accusé d'avoir largement repris, dans son livre Ticket d'entrée (Grasset, 2011), des passages de la traduction française de American rigolos : chroniques d'un grand pays (2003, Payot et Rivages) de Bill Bryson, sans le citer et en maquillant grossièrement l'emprunt par une substitution de mots ou en pratiquant le copier-coller.

La notion d'intertextualité bénéficie d'une multiplicité d'origines et d'approches. Il en résulte que ses définitions et ses pratiques sont également diverses. Introduite dans les années 1960 par Julia Kristeva, théorisée auparavant par Mikaïl Bakhtine sous le terme de «dialogisme», l'intertextualité renvoie à une conception du texte comme lieu d'une interaction complexe avec les différents textes qui lui préexistent et qui forment un système textuel composant le champ dans lequel s'opère l'écriture.

S'il est naturel que l'écrivain se réfère au monde littéraire qui l'entoure et aux lectures qu'il a pu faire, du point de vue du droit, l'argument de l'intertextualité, soulevé en défense à une accusation de plagiat, n'a pas de valeur juridique. À ceux qui invoquent le cas de La Fontaine, multipliant les emprunts à Montaigne, Ésope ou Phèdre pour écrire ses fables, il faut répondre que les pratiques du XVII ${ }^{\mathrm{e}}$ siècle ne peuvent définitivement plus servir de modèle ni d'excuse car il existe aujourd'hui des textes de loi qui structurent clairement le droit d'auteur.

Le Code de la propriété intellectuelle (CPI) dispose que «l'auteur d'une œuvre de l'esprit jouit sur cette œuvre, du seul fait de sa création, d'un droit de propriété incorporelle exclusif et opposable à tous ».

Une œuvre se verra protégée si elle est originale. L'originalité est la marque de la sensibilité de l'auteur, la traduction de sa perception personnelle du sujet qu'il traite : elle est constituée par les choix qu'il a effectués dans sa rédaction et qui n'étaient pas imposés par ce sujet.

Juridiquement, l'originalité n'est ni l'inventivité ni la nouveauté. Au regard de la loi, une œuvre peut être originale sans être nouvelle : elle bénéficiera donc de la protection du droit d'auteur, même si elle reprend, à sa manière, un thème cent fois exploré. De même, une œuvre peut aussi être originale tout en devant sa contribution à une autre œuvre. Il en est ainsi des traductions, adaptations, transpositions, etc.

1. AFP, «Accusé de plagiat, Joseph Macé-Scaron affine sa défense», Libération, 23 août 2011. 
En matière littéraire, l'originalité d'un texte, pour un juriste, se retrouve dans deux éléments : la composition et l'expression. Ce sont ces deux éléments qui seront pris en considération pour mesurer juridiquement si l'originalité du texte a fait ou non l'objet d'une contrefaçon. La composition est l'ordonnancement des chapitres, le déroulement de la trame, la mouture, le plan, la disposition des idées : elle résulte notamment de ce que la critique génétique appelle la structuration, le programme, le scénarique. L'expression, c'est le lexique et la syntaxe de l'écrivain, le choix des mots et des tournures de phrase : ce dont l'analyse des manuscrits suit l'émergence et l'évolution à travers le processus de textualisation, en particulier au cours des phases rédactionnelle et pré-éditoriale. Composition et expression correspondent à ce que la génétique des textes désigne par le terme d'endogenèse : non pas un processus d'appropriation du déjà-écrit comme dans le cas de l'exogenèse, mais un processus où l'écriture se trouve centrée sur ses propres moyens formels et imaginaires, sans devoir la réalisation du texte à aucun emprunt caractérisé, par les seules ressources du travail de l'écrivain qui crée une forme écrite personnelle pour exprimer une idée. C'est cette originalité que la loi protège en premier lieu.

En revanche, la loi exclut de son champ de protection l'opposé de la création de forme : l'idée. On a coutume de dire que «les idées sont de libre parcours ${ }^{2}$ ».

\section{Le droit d'emprunter}

Ainsi, toute œuvre qui répond aux critères exigés pour une protection par le droit d'auteur ne peut être reproduite ou représentée, en tout ou partie, qu'avec l'autorisation du titulaire des droits sur l'œuvre. Mais la loi, renforcée par la jurisprudence, révèle de véritables brèches dans le droit d'auteur, qui permettent à des tiers, sous certaines conditions, de procéder sous une forme ou une autre à l'exploitation de l'œuvre.

Le droit français tente de trouver un équilibre en privilégiant l'intérêt des auteurs, en leur accordant un droit exclusif, tout en ménageant l'intérêt du public par des mécanismes d'exception à ce droit. Le CPI énumère les situations dans lesquelles les tiers peuvent se passer de toute autorisation du titulaire des droits : représentations privées, copies privées, citations, analyses, revues de presse, discours officiels et politiques, textes légaux, décisions judiciaires et hymnes nationaux, parodies, pastiches et caricatures.

Pour déterminer si une citation échappe aux obligations d'autorisation définies par le droit de reproduction, il faut s'attacher à identifier le but particulier qu'elle cherche à atteindre et qui pourrait l'en exempter : finalité critique, polémique, pédagogique, scientifique ou d'information. En ce sens, la reproduction de citations reste donc fortement ouverte.

En sus d'un but non commercial et d'une finalité particulière acceptable, la citation doit être «courte». Cette brièveté s'apprécie non seulement par rapport à l'œuvre de départ (il ne faut pas reproduire une part trop importante de l'œuvre citée) mais aussi par rapport à l'œuvre d'arrivée (il n'est pas possible de ne constituer son ouvrage que de citations). C'est ainsi qu'une trentaine de citations rassemblées dans une plaquette seront considérées comme une contrefaçon, alors que les mêmes citations intégrées à une étude de deux cents pages bénéficieront de l'exception légale. Il n'existe en réalité pas de barème précis permettant de déterminer à coup sûr ce qui relève du droit de citation et ce qui appartient à la contrefaçon.

2. Henri Desbois, Le Droit d'auteur en France, Paris, Dalloz, 3e éd., 1978, no 17 sq. 
Enfin, l'auteur, pour exciper de l'exception de citation (pour se prévaloir du droit de citer), doit respecter le droit moral de l'auteur. Il doit démontrer le respect de l'esprit de l'œuvre dont la citation est tirée, mais également de sa forme, etc. Il est bien évident que seules les véritables dénaturations sont répréhensibles, c'est-à-dire celles qui ont tendance à donner une vision fausse de l'œuvre citée. De même, le citateur ne doit pas oublier de mentionner sa source - il s'agit là expressément d'une obligation légale, qui découle par surcroît du droit au respect qui est dû au nom de l'auteur de l'œuvre citée.

Dès lors que la loi s'applique à encadrer le droit de citation, tout emprunt non conforme aux prescriptions légales, que l'emprunteur tentera de défendre en arguant d'intertextualité, reçoit une qualification bien précise en droit : la contrefaçon.

Les spécialistes du droit d'auteur utiliseront en effet le terme de «contrefaçon» là où les éditeurs et leurs auteurs parlent de «piratage», de «pillage», de «plagiat» ou encore de «violations des contrats». Le mot «plagiat», qui n'appartient pas au vocabulaire juridique, accompagne des procès médiatiques et défraye souvent la chronique. Ce terme appartient davantage au registre moral : il est employé pour stigmatiser un emprunt que la morale réprouve mais qui, du point de vue du droit, appartient à la catégorie plus vaste de la contrefaçon.

Quelle que soit la variété des pratiques dénoncées, la contrefaçon et sa répression répondent à un régime juridique précis. La contrefaçon peut se définir comme «le fait d'utiliser publiquement, de quelque façon que ce soit, sans avoir requis son consentement préalable, l'œuvre d'autrui, faisant l'objet d'une protection accordée par la loi ${ }^{3}$ ». Ce délit concerne donc n'importe quelle création protégée par le droit d'auteur : le texte en lui-même, mais aussi le titre du livre, sa composition, la maquette de sa couverture, le dessin placé en frontispice, les éléments du cahier photo, etc.

Toutes catégories confondues, les rhétoriques juridiques que développent plagiaires et plagiés devant les tribunaux sont désormais bien rodées.

\section{Le rôle du tableau comparatif}

Parmi les «emprunteurs», il reste aujourd'hui des adeptes du simple copier-coller, immédiatement perceptible. Néanmoins, des formes d'emprunts plus sophistiquées se multiplient et se révèlent moins faciles à déceler. Les possibilités d'emprunt, à l'ère du numérique, sont maximisées.

En pratique, les professionnels du droit - avocats et magistrats - s'aident tous d'un véritable tableau comparatif des deux œuvres en cause. C'est alors une fastidieuse quête des «points de contact ${ }^{4} »$. Les juges vont en effet chercher si le sujet choisi (la violence familiale, par exemple) nécessite le recours à des situations ou des expressions communes. Il faudra également faire la part de ce qui appartient au fonds commun de la littérature (par exemple, l'amour impossible sur fond de clans ennemis). Le plagiat peut aussi bien porter sur la «composition» de l'ouvrage (c'est-à-dire le scénario) que sur l' 'expression» (c'est-à-dire

\section{La rhétorique judiciaire des plagiaires et des plagiés}


le style, la formulation). La contrefaçon s'appréciant par les ressemblances et non par les différences, quelques similitudes suffisent à caractériser la contrefaçon et à entraîner la condamnation.

\section{La défense du plagiaire}

Le plagiaire va s'efforcer de souligner les différences fondamentales entre les deux traitements du sujet. Pour le reste - c'est-à-dire les ressemblances - il va d'abord recourir à la notion de fonds commun. Cette rhétorique le conduit souvent à se réfugier sous la bienheureuse notion de hasard («la faute à pas de chance»), qui défie les lois sur la propriété intellectuelle comme de la statistique, en vertu de laquelle les chances se mesurent à l'aune de plusieurs millions de probabilités.

Les armes du plagié sont alors simples. Si le sujet des ouvrages litigieux est assez convenu, le plagié va s'acharner à démontrer l'extrême originalité de sa vision.

Rabaisser littérairement le plagié reste encore une ruse qui n'a pas fait ses preuves. L'écrivaine Calixthe Beyala, accusée à deux reprises de "plagiat», a eu plusieurs fois l'occasion de resservir la même interrogation : est-ce que quelques lignes suffisent à faire une œuvre?

Plus élégante est la formule de Patrice Delbourg, autre auteur épinglé, selon laquelle l'emprunt est forcément un hommage.

C'est encore la reprise inconsciente de souvenirs littéraires que va d'ailleurs invoquer le plagiaire. Il s'agit cependant là d'un exercice périlleux puisqu'il consiste implicitement à glorifier aux yeux des juges ce même plagié qui vous poursuit en justice... Invoquer l'hommage à l'auteur plagié n'est d'aucune utilité devant un magistrat s'il ne s'agit pas d'un emprunt s'appuyant clairement sur l'autorité intellectuelle d'un travail antérieur qui se trouve cité explicitement, notes de bas de page comprises.

L'appel à l'erreur légitime (oubli, bibliographie avalée par l'ordinateur, etc.) reste néanmoins une technique de défense sobre mais souvent réductrice du montant des dommages-intérêts.

Quant à l'argument de l'intertextualité, invoqué par Joseph Macé-Scaron, qui voudrait que le texte littéraire se constitue nécessairement de transformations et de combinaisons de différents textes antérieurs, appelés à entrer en interaction, est un argument littéraire et non juridique.

\section{Le plagiat hissé au rang des beaux-arts}

Le plagiat est aussi parfois évoqué comme un genre incontournable. Le plaideur citera alors opportunément Giraudoux, qui avait déclaré que le plagiat était à la base de toute littérature.

Il est même apparu utile à certains - dont la main était malencontreusement prise dans un sac - de hisser le plagiat au rang des beaux-arts. La référence aux grands écrivains plagiaires - tels que recensés dans l'indispensable Dictionnaire des plagiaires de Roland de Chaudenay - va contribuer non seulement à la défense du plagiaire mais également à sa thérapie et à son exercice d'autopromotion 5 . 


\section{Débat judiciaire et débat public : plagié et plagiaire face à la diffamation}

Le plagiaire va parfois en arriver à démontrer que le plagié a lui-même été plagiaire...

D'ailleurs, apparaissent enfin d'intéressantes mises en abîme lorsque le plagiaire ou le plagié vont intenter tous azimuts des procès en diffamation, dont l'enjeu va porter sur le bien-fondé ou l'issue du procès pour plagiat. Le plagiaire devient alors à son tour un redoutable procédurier. C'est ainsi que celui qui est accusé de «plagiat» en appelle parfois à la précision juridique. Les spécialistes du droit d'auteur lui préfèrent, comme on l'a vu, le terme de «contrefaçon», considérant que le terme de «plagiat» stricto sensu devrait être réservé à l'exploitation d'une œuvre par un auteur assez habile pour ne pas être juridiquement répréhensible 6 ...

6. André Bertrand, «La protection de la composition en matière d'œuvres littéraires et audiovisuelles», Cahiers du droit d'auteur, 1990, no 27, p. 17. Isabelle Bureau, «L'intrigue : le passage de l'idée à son expression», Les Cahiers de propriété intellectuelle, octobre 1994, vol. 7, n 1, p. 51 (article sur le droit canadien). 
Emmanuel Pierrat est avocat au barreau de Paris. Spécialisé en droit de la propriété intellectuelle, il est l'auteur, parmi de nombreux ouvrages, de L'Auteur, ses droits et ses devoirs (Gallimard, coll. «Folio essais », 2020) et du Droit d'auteur : un dispositif de protection des æeuvres (Le Lombard, 2016).

\section{Intertextualité et exogenèse devant les tribunaux}

Le présent article est une réflexion, au regard du Code de la propriété intellectuelle, sur la question de la contrefaçon littéraire. Si les circulations intertextuelles tendent à inscrire les œuvres littéraires dans un vaste réseau, dans quelle mesure le droit encadre-t-il la démarche créatrice des écrivains ? À partir de quand cette dernière, souvent innervée par des éléments exogènes dont témoignent les documents de genèse, tombe-t-elle sous le coup de la loi?

This article discusses the question of copyright infringement and intellectual property law. If practices of intertextual circulation tend to implicate literary works in a vast network, to what extent can law delimit the creative process of writers? Specifically, when does the creative process, often frustrated by exogenetic elements during the drafting stages, fall under the purview of the law?

Dieser Beitrag versteht sich als eine Reflexion über die Frage der literarischen Fälschung im Hinblick auf das Gesetz über geistiges Eigentum. Wenn die intertextuelle Zirkulation dazu neigt, literarische Werke in ein riesiges Netzwerk einzubinden, inwieweit rahmt dann das Gesetz den Schaffensprozess der Schriftsteller ein? Ab wann ist der kreative Prozess, der oft durch exogene Elemente belebt wird, wie die Dokumente der Genese belegen, gesetzlich geregelt?
El presente artículo constituye una reflexión - teniendo en cuenta el Código de la Propiedad Intelectual - acerca de la cuestión de la imitación en literatura. Si la circulación intertextual tiende a inscribir las obras literarias en una vasta red, ¿en qué medida el Derecho puede encuadrar el proceso creativo de los escritores? ¿A partir de qué momento este último - a menudo inervado por elementos exógenos de los que dan cuenta los documentos de génesis - puede ser sancionado por la ley?

Este artigo é uma reflexão, no âmbito do Código da propriedade intelectual, sobre a questão da contrafação literária. Se as circulações intertextuais tendem a inscrever as obras literárias numa vasta rede, em que medida o direito enquadra o processo criativo dos escritores? A partir de quando é que este último, frequentemente alimentado por elementos exogenéticos como testemunham os documentos da génese, cai sob a alçada da lei?

$\mathbf{L}$ 'articolo seguente è una riflessione, dal punto di vista del Codice della proprietà intellettuale, sulla questione della contraffazione letteraria. Se le circolazioni intertestuali tendono a iscrivere le opere letterarie in una vasta rete, in quale misura il diritto inquadra la pratica creatrice degli scrittori? Da quando quest'ultima, spesso innervata da elementi esogeni rivelati dai documenti genetici, è sottoposta alla legge? 